\title{
Post-operative shoulder imbalance in adolescent idiopathic scoliosis: a study of clinical photographs
}

K. Venugopal Menon ${ }^{1 *}$, Haroon M. Pillay ${ }^{2}$, Anbuselvam $\mathrm{M}^{2}$, Naveen Tahasildar ${ }^{3}$ and Renjit Kumar $\mathrm{J}^{4}$

\begin{abstract}
Study design: Retrospective observational study.

Objective: To assess what features determine post-operative shoulder asymmetry in Adolescent Idiopathic Scoliosis (AIS).

Summary of background data: Shoulder balance is one of the major determinants of the cosmetic outcomes of AIS surgery. Yet, other than level of the shoulders we are not clear what parameters are to be measured to assess torso symmetry. This study looks at the various features that might affect the appearance of the shoulder region.

Methods: The records of 157 operated cases of AIS were retrospectively reviewed. Eight patients with documented post-operative shoulder asymmetry and were dissatisfied with their cosmetic outcomes were selected for the study. Their clinical photographs alone were studied. Three regions- the base of the neck, the shoulder and upper arm region- were analysed separately. Four measures each for the neck and shoulder and two for the arms were documented. No statistical tools were employed since the numbers were quite small but consensus was obtained between two Consultant Orthopaedic surgeons regarding the cosmetic impact of each parameter.

Results: The neck and the shoulder appeared independent determinants of cosmesis of the proximal trunk. The base of neck symmetry seemed to be dependent on four features viz. centralization of the neck, neck tilt, trapezius angle and base of neck angle. The appearance of the shoulder itself depended on its level, axillary fold level, scapular level and the scapular prominence. The upper arm parameters appeared less critical in determining the cosmetic impact.

Conclusions: Proximal trunk symmetry in AIS depends on the symmetry of the base of the neck and shoulder regions. The level of the shoulders, axillary folds along with the base of neck angle, Trapezius angle appear to be key determinants of symmetry.
\end{abstract}

Keywords: Adolescent Idiopathic Scoliosis, Shoulder balance, Clinical photograph, Torso symmetry, Trunk balance, Neck symmetry

\section{Background}

The overwhelming impact of evidence based medical practice is manifest by the need to describe and measure clinical parameters in detail-even qualitative data like satisfaction or cosmetic appearance needs to be defined and measured with precision [1]. In Adolescent Idiopathic Scoliosis (AIS) trunk symmetry, sagittal and coronal plane balance and shoulder balance are the features that are accepted as essential cosmetic considerations [2]. While

\footnotetext{
* Correspondence: kvm422@hotmail.com

'Orthopaedics, Khoula Hospital, Mina al Fahal, Muscat, Sultanate of Oman

Full list of author information is available at the end of the article
}

there is uniform agreement that shoulder balance is of paramount importance, (Fig. 1) there is little consensus on what constitutes optimum shoulder symmetry in AIS patients. Traditionally the shoulder levels were measured by one of many methods radiologically [2-4]. Numerous radiological parameters have been described and their relative value in measuring shoulder balance compared including T1 vertebral tilt, Clavicle angle, Coracoid process height difference, Trapezius length, First Rib -Clavicle height, Clavicle rib cage intersection difference, First Rib angle, Clavicle tilt angle difference, Radiographic shoulder height etc. [5-10] Unfortunately it has been established 


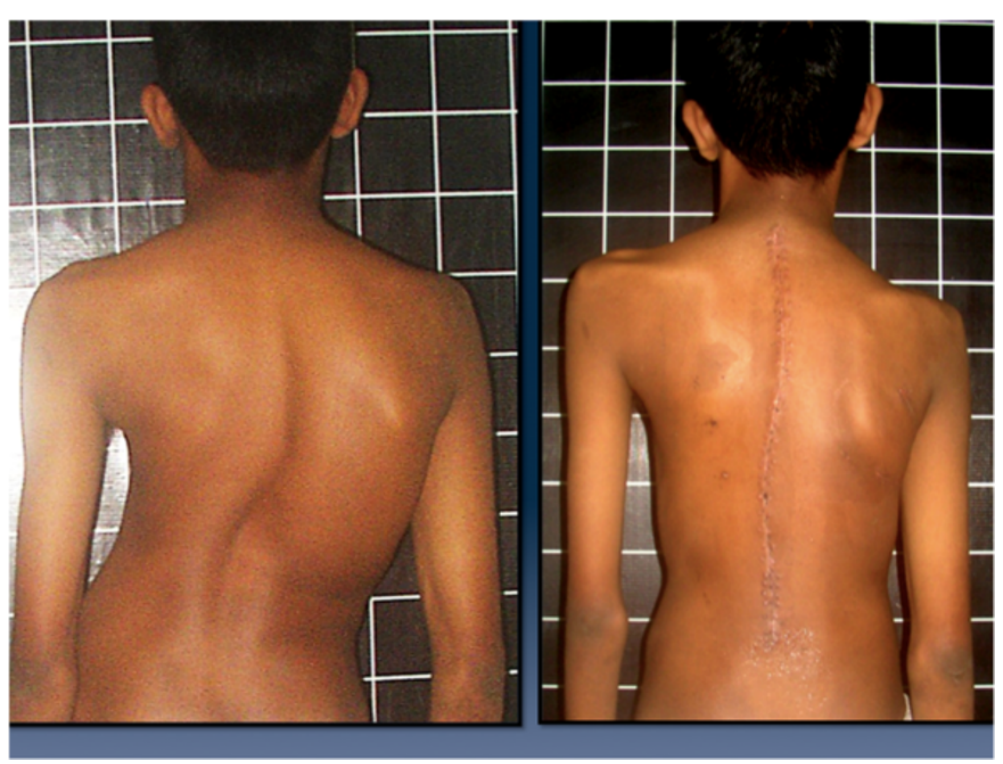

Fig. 1 Pre and post-operative images of a patient of AIS. Note that despite the poor trunk alignment in the pre-op state the shoulders are balanced and after surgery though the trunk alignment has improved the shoulder has become unbalanced. In this patient the base of the neck features appear relatively preserved compared to the shoulder level

that clinical shoulder balance or the absence of it does not always correlate well with radiological imbalance $[2,11,12]$. Winter in 1989 suggested that shoulder elevation, trapezial fullness and left thoracic rib prominence might be the clinical features of shoulder asymmetry seen in double thoracic curves [13]. Qui and co workers [11] were perhaps the first to point out the significance of clinical features of the proximal trunk imbalance and how to measure them on digital photographs. They have used pre-operative clinical photographs to determine areas of symmetry between the sides of the torso. Based on clinical photographs taken from posterior aspect, these authors also suggested that radiological balance does not always coincide with clinical symmetry of the shoulder. The research question then is what precise features are responsible for the unsightly appearance of the shoulder region in operated cases of AIS with shoulder imbalance?

Cosmetic disfigurement in AIS is a major concern for patients and their parents [14] and it can be studied under several domains. The three most important ones that have been documented well are radiological measures of spine and trunk alignment (Cobb angle, Apical vertebral translation, spinal balance etc.), patient or parent perception of trunk appearance and body image, (Walter Reed Visual Analogue Scale-WRVAS, Spinal Assessment Questionnaire-SAQ etc.) and objective assessment of trunk symmetry on clinical photography or surface topography $[15,16]$. These domains may be summarized as Radiological, Patient Satisfaction and
Clinical domains. The idea of objective measurement of a subjective perception- cosmetic disfigurement- is not novel. Moire's surface topography, ISIS scan, Quantec, Jenoptik Formetric, Raster photography etc. are but some of these [17-21]. Several authors have tried to introduce unprejudiced observational criteria into describing deformity $[17,20]$. Clinical photography and videography have also been used for this purpose. The Trunk Aesthetic Clinical Evaluation is one such tool that uses clinical photographs for measuring cosmetic dysfunction where in addition to shoulder and scapular dissymmetry the waist and thoracic cage are taken into consideration [22]. Newer devices have also been described like the $\mathrm{ISIS}_{2}$. While these tools are typically used to determine the degree of disfigurement and its correction, specific measures of what constitutes right vs. left asymmetry of the torso, has not been clearly addressed.

This paper is an attempt to unveil what elements might be responsible for torso symmetry and therefore asymmetry after AIS surgery; hopefully this knowledge will help us better measure it and prevent or treat it.

\section{Materials and methods}

The records of 157 operated cases of Adolescent Idiopathic scoliosis cases from two centers in Asia were reviewed retrospectively for this study. All of them had pre and post-operative clinical photographs that were available for review. There were 131 females and 26 males in the group and their mean age was 14.55 years. In the post operative follow-up records it was documented that eight patients had significant shoulder 
imbalance and were unhappy about their appearance. This included patients who were subjectively concerned about the appearance of their shoulders as well as cases where the clinician had recorded objective shoulder asymmetry (see Table 1). Four of them were boys and four were girls with a mean age of 15.06 years and an SD of 1.266. The clinical photographs of these eight cases formed the core materials for this study. All the post-operative photographs were taken at the 3 months review after surgery. Only the post-operative photographs were analysed for their cosmetic impact. In both centers clinical photographs were taken with a point and shoot digital camera at fixed distance of two meters. The camera was fixed at the level of the scapulae and focused at the base of the inter-scapular region. The patient stood relaxed against a blank wall or a grid (which was the standard practice in one of the institutions.) The footprints were marked on the floor for maximum reproducibility. The arms were held relaxed at the sides. Appropriate dress with tied up hair allowed maximum visibility of the neck and upper trunk without violating the patient's cultural sensitivities (one patient with significant shoulder asymmetry but did not have an optimum post operative photograph is seen in Fig. 5). Only one standing image of the back view of the trunk was utilized for the purpose of this study.

At one of the two institutions the Hospital information system had the capability to make angular, linear and area measurements on images and the measures were done digitally. At the other establishment the digital photographs were downloaded on to either a PowerPoint or Sketchbook Express page and the lower half of the body cropped out. Printouts were obtained and on these paired points were marked on the tips of the acromion, axillary folds, inferior poles of the scapulae, neck to Trapezius junction (inflexion point), and the center of the neck about the $\mathrm{C} 7$ spinous process (Fig. 2) The following lines and angles were measured on all the eight images manually-

- Inter acromial line and angle with the horizontal. The line joining the points of the acromion were marked (light blue arrows in Fig. 2) and the angle with the horizontal measured.

- Inter axillary line and angle with the horizontal. The axillary folds were marked (grey arrows in Fig. 2) and the angle measured between the connecting line and the horizontal.

- Inferior poles of the scapulae connecting line and angle with the horizontal. White arrows in Fig. 2. illustrates the marking of these points.

- Differential prominence of the inferior scapular poles (documented as yes or No). See Figs. 2, 3, 4.
- Base of the neck line and angle with the horizontal. Fig. 2 illustrates how the inflexion angle of the neck is marked and Fig. 4 shows how the angle is measured.

- Neck- Trapezius angle difference between the right and left. The angle at the inflexion point of the neck-Trapezius junction is measured and the difference documented (see Fig. 4).

- Neck inclination angle from the vertical. This is essentially the long axis of the neck against the vertical axis shown in Fig. 3. The axis of the neck is drawn by taking any two transverse diameters and joining their midpoints.

- Neck centralization- difference in the distance between the outer points of the shoulders to the center of the neck at C7 (expressed as percentage of the total distance between the shoulders). See white arrows in Fig. 3.

- Arm width difference- difference between arm width at the axillary fold level (expressed as percentage of the total arm width). Fig. 3.

- Arm drop angle- difference in angle between upper arm and vertical axis on either side (abduction is recorded as a positive value and adduction as a negative one) Fig. 3.

The final values from this series are presented in a tabular form in Table 1. Two clinicians evaluated all the eight images and decided where the aesthetic problem lies- shoulder, neck base, or upper arm or a combination of these. No statistical tools were used- only a consensus between the observers was obtained and documented in Table 1. The measures corresponding to major disfigurement were then identified from the table.

Figure 1 depicts a patient of AIS with post-operative shoulder imbalance despite excellent correction of the trunk deformity. Figure 2 illustrates the various points, lines and angles that are measured for this assessment of the shoulder domain while Fig. 3 demonstrates the "Arm" features as well as the neck tilt and neck centralization concept. Figure 4 clearly documents the base of neck angle and the Trapezius angle difference that were used in this study.

\section{Results}

The results of this study are summarized in Table 1. Six of the eight patients recorded here displayed dissatisfaction with the appearance of their shoulder region while two were not aware of major aesthetic problems but the surgeon who reviewed them during follow up documented visible anomaly. By consensus between the two evaluating surgeons four patients had predominantly shoulder imbalance, two each had neck base and combined neck and shoulder imbalance. The arm features 
Table 1 The eight cases and the various measures recorded are depicted. Angles are measured in degrees and lengths are expressed as percentage of the total length. Major aesthetic disfigurements are highlighted in pink

\begin{tabular}{|c|c|c|c|c|c|c|c|c|c|c|c|c|c|c|c|}
\hline SI No. & Name & Age & Gender & Neck tilt Le & Neck base Le & Trap Le & Neck center diff & Sh level & Ax level & Scap level & Scap prom & Arm width diff & Arm drop Le & clinical impression & Who noticed \\
\hline 1 & MIZ & 16 & M & 0 & 12 & 12 & 0.044 & 6 & 2 & na & na & 0 & 10 & neck, sho & Dr \\
\hline 2 & JT & 15.5 & M & 2 & 18 & 10 & $4.40 \%$ & 3 & 1 & 2 & yes & $7.70 \%$ & 8 & Neck & $\mathrm{Pt} / \mathrm{Dr}$ \\
\hline 3 & A & 14 & M & 5 & 7 & 5 & $3.64 \%$ & 10 & 4 & 15 & yes & $18.50 \%$ & 0 & Shoulder & $\mathrm{Pt} / \mathrm{Dr}$ \\
\hline 4 & NMMJ & 14.5 & $\mathrm{~F}$ & na & na & na & na & 6 & 3 & 0 & 0 & $9.10 \%$ & 10 & shoulder & $\mathrm{Pt} / \mathrm{Dr}$ \\
\hline 5 & $\mathrm{RH}$ & 14.5 & M & 3 & 6 & 9 & $4.76 \%$ & 5 & 4 & 0 & 0 & 0 & 2 & Neck & Dr \\
\hline 6 & FR & 13.5 & $\mathrm{~F}$ & na & na & na & na & 7 & 5 & 1 & Yes & $25 \%$ & 0 & shoulder & $\mathrm{Pt} / \mathrm{Dr}$ \\
\hline 7 & AJ & 17.5 & $\mathrm{~F}$ & 0 & 7 & 8 & 0.05 & 8 & 5 & 6 & 0 & $21.21 \%$ & 1 & shoulder & $\mathrm{Pt} / \mathrm{Dr}$ \\
\hline 8 & SS & 15 & $\mathrm{~F}$ & 2 & 9 & 4 & 0 & 4 & 3 & 9 & yes & $6.67 \%$ & 0 & neck, sho & $\mathrm{Pt} / \mathrm{Dr}$ \\
\hline Mean & & 15.063 & & & & & & & & & & & & & \\
\hline SD & & 1.27 & & & & & & & & & & & & & \\
\hline
\end{tabular}

Legends to Table: 1

Column 5: Neck Tilt Angle

Column 6: Neck Base Angle

Column 7: Trapezius angle difference

Column 8: Neck Center difference

Column 9: Shoulder level

Column 10: Axillary level

Column 11: Scapular level

Column 13: Arm Width Difference

Column 14: Arm drop Angle

Column 15: depicts the 2 observer's impression of the clinical photograph

The bold faced text suggests the measures for clinically significant disfigurement as assessed bycolumnn 15 of the table. However please note that these are not statistically tested 


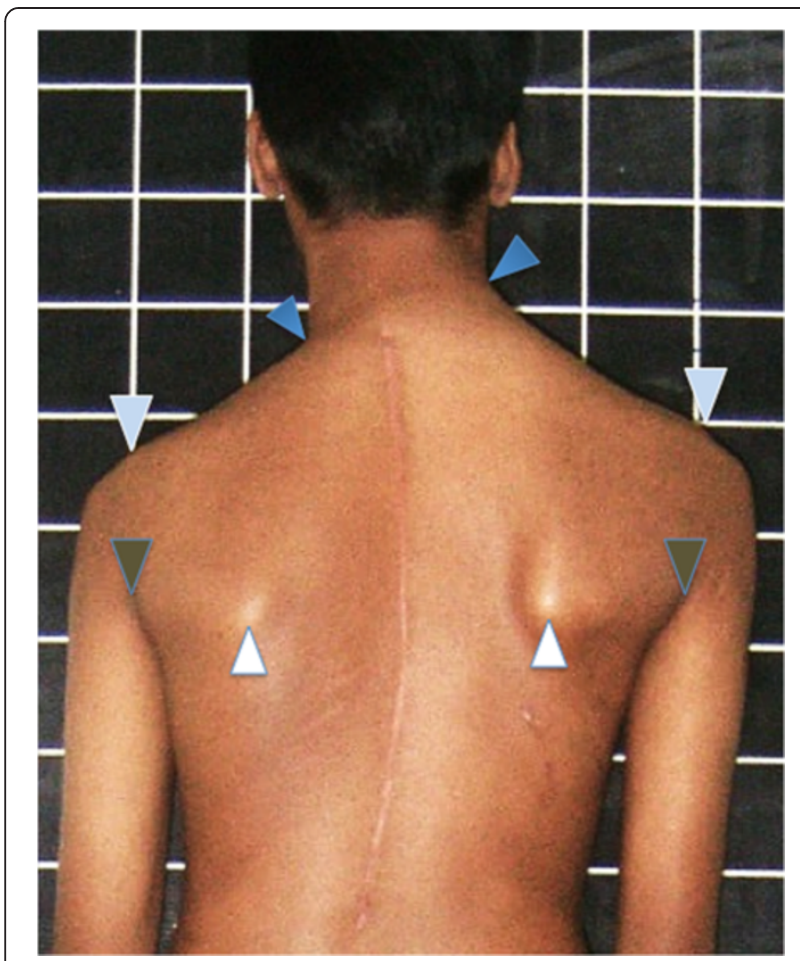

Fig. 2 Post operative shoulder imbalance demonstrating the marking of the neck inflexion points (Dark Blue arrows), points of the shoulder (acromion) (Light Blue arrows), axillary folds (Grey arrows), and scapular prominence at its inferior poles (White arrows). In this case the base of the neck appears significantly tilted compared to the shoulder level itself

did not appear (to the observers) to make a major impact in the cases studied though some of the cases did have significant measured disparity (Cases 1,4 in terms of arm drop angles and cases 3 and 7 with regard to the arm width difference). It was observed that each region is independently capable of creating a perception of asymmetry though often deformities in both regions coexist. For example the child in Fig. 2 demonstrates reasonable shoulder alignment but grossly distorted neck features. Similarly the patient depicted in Fig. 1 shows predominantly shoulder level dissymmetry affecting the acromial, axillary and scapular lines. Incidentally, this case also has asymmetric Trapezial angles and eccentric placement of the neck on the shoulders aggravating the cosmetic disfigurement.

The major disfigurement in the neck appeared to be inclination of the neck from the vertical axis (Fig. 2) but this was only an apparent phenomenon due to the base of neck being inclined. Though no statistical tools were employed due to the small number of cases in this study, from Table 1 it appears that neck base angle over $9^{0}$ as well as Trapezius angle difference over $9^{0}$ seem to correlate with significant unsightliness. (The power of the

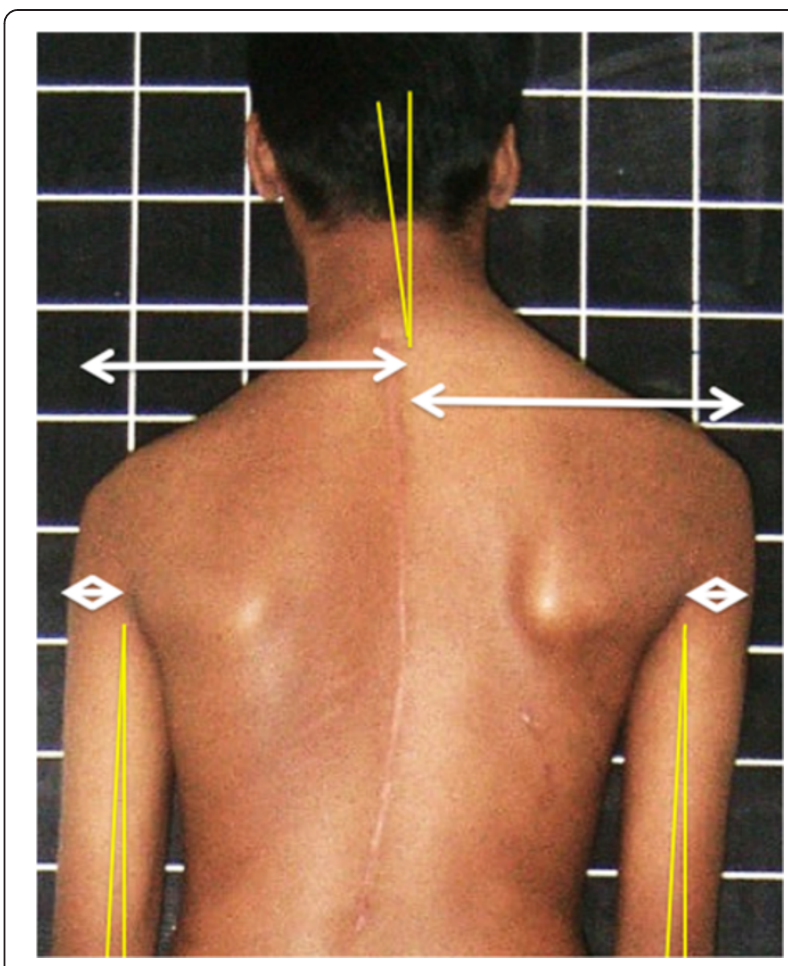

Fig. 3 Illustrates the technique of measuring the axis of the neck in relation to the vertical axis and the centralization of the neck on the shoulders; additionally the width of the arm at the axillary fold level and the angle between the arm and vertical axis are marked out

study did not allow cutoff values for each measure to be determined based on the data presented.) The centrality of the neck was measured by the distance from the edge of the shoulder to the center of the neck on either side. This parameter was noted to be abnormal frequently (Table 1) though less aesthetically damaging than other features. The difference in Trapezius angles on either side appeared to contribute substantially to visualized deformity. This was often better seen in boys than in girls and in thin children.

In the shoulder itself major contributions to asymmetry were by the shoulder level and to a lesser extent by the axillary fold level and scapular prominence. A shoulder level angle of $6^{\circ}$ and a scapular angle of $9^{\circ}$ appeared to be the threshold for aesthetic impact though again not statistically validated due to small numbers. The level of the axillary fold, its shape and contribution to the body contour impacts the overall appearance though in this study it had a lesser impact than shoulder level. Prominent scapular poles were seen equally in patients with significant shoulder asymmetry as well as those without making the contribution of this feature less reliable than imagined. When assessing the axillary fold it was observed that the width of the upper arm seemed to be different on both sides suggesting that 


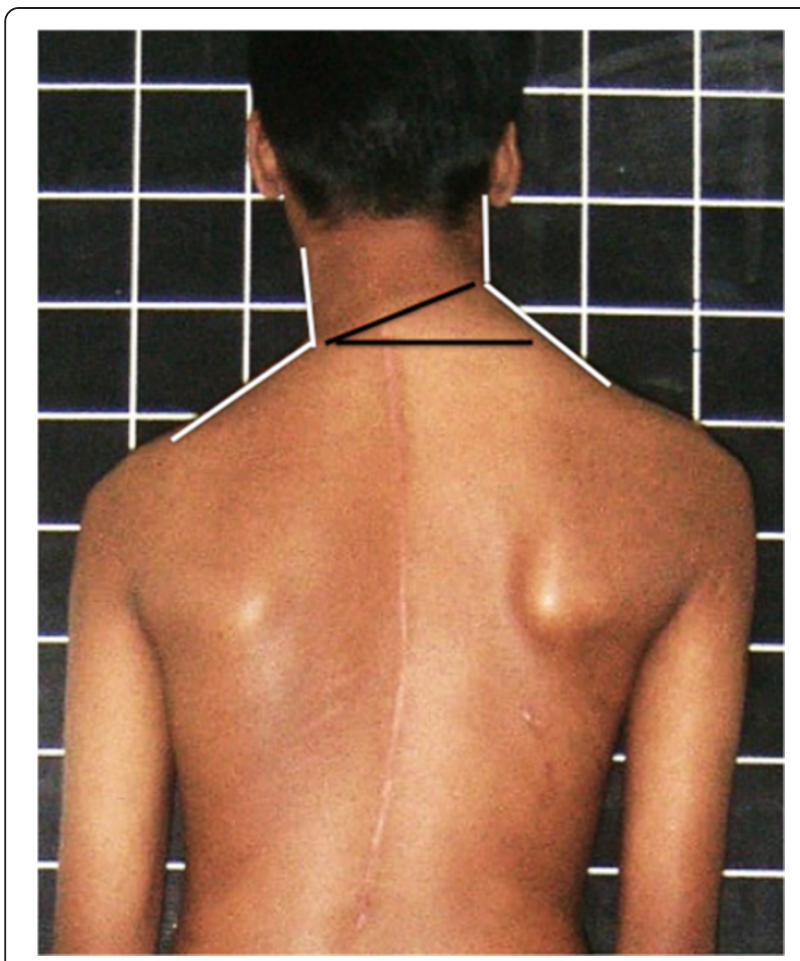

Fig. 4 The Neck-Trapezius angle (white lines) is marked demonstrating a clear difference between the sides as well as the angle of the base of the neck (black lines) from the horizontal plane. Please note that the neck base appears significantly more tilted than the shoulders and axillary folds in this patient

there might be an element of trunk rotation that affects the appearance of the torso as well (Fig. 5). The arm width and the long axis of the upper arm appeared to have a much lesser impact on appearance despite the measured difference between sides.
The influence of each component of the deformity on the overall unsightliness is variable in each given case. Moreover, in each patient there are combinations of elements that contribute significantly and others that matter a little less to the general disfigurement. Perhaps there are particularly unpleasant combinations- in this case series the numbers were too small to analyse all these factors. There is also a subjective element to the cosmetic influence of each component of the deformity; again in this study neutral observers were not employed for the assessment of impact of each feature.

\section{Discussion}

Shoulder balance has long been recognized as a major contributor to the overall trunk appearance after scoliosis surgery. When a right thoracic curve with a high right shoulder was operatively corrected most often the shoulder balanced itself post operatively. The discovery of the double thoracic curve and the left shoulder elevation introduced confusion in the instrumentation strategy. A lot of emphasis was initially placed on the proximal thoracic curve and its role in determining the level of the shoulder. Most authors have reported that structural Proximal Thoracic (PT) curve would result in the left shoulder being elevated and nonstructural ones would lead to right shoulder elevation [23]. The criteria for structurality of curves varied between authors. As a natural consequence a lot of literature emerged on selecting Upper Instrumented Vertebra (UIV) to achieve balanced shoulders [8-10].

Simultaneously several authors started looking at the radiographic criteria to define shoulder balance [24]. Since it was soon established that the T1 vertebral tilt traditionally used to bench mark shoulder level was not

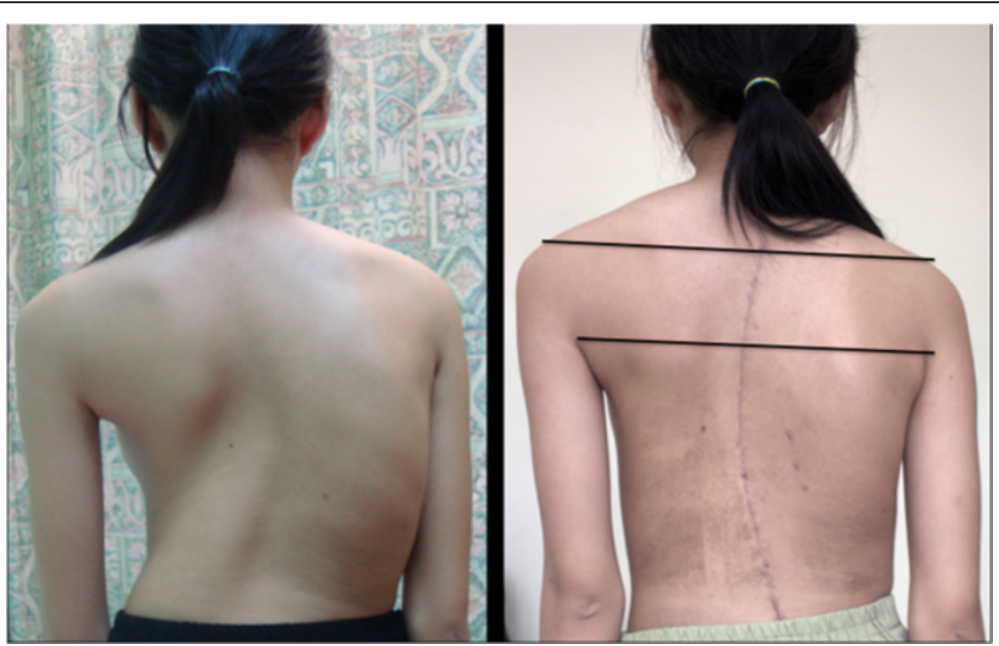

Fig. 5 Figure depicts a patient with post-operative shoulder imbalance. However, the base of the neck is obscured by the hair though shoulder and axillary levels are clearly visible 
a reliable indicator of clinical shoulder level, multiple other measures like Clavicle angle, Coracoid process height difference, Trapezius length, First Rib -Clavicle height, Clavicle rib cage intersection difference, First Rib angle, Clavicle tilt angle, Clavicle chest cage angle difference, First Rib Index etc. emerged [25, 26]. In recent years it has become increasingly apparent that radiological parameters of shoulder level do not always coincide with clinical levels and that the patients' subjective perception of body image has little to do with the $\mathrm{x}$-ray appearance [2]. Along with the SRS outcome tools a number of other patient satisfaction measures were developed. The Walter Reed Visual Assessment Scale was one of the earliest and most comprehensive [27]. It did estimate the patient's perception of shoulder balance in two domains- shoulder levels and scapular prominence. Though there is no numerical description of the five grades in each domain and it looks at the posterior aspect of the body only, it was a major contributor to our understanding of shoulder symmetry in AIS. The authors later upgraded the questionnaire eliminating the scapular prominence and incorporating a lateral view instead (SAQ) [28, 29]. Bago and colleagues introduced the Trunk Appearance Perception Scale (TAPS) questionnaire and reported good inter-observer reliability using this system [30]. The shoulder, trunk and waist as seen from the back were objectively studied by Zaina et al. [22] to formulate the TRACE tool which has been found to be fairly reproducible by the authors. But the question remained- what makes the patient's shoulder look unsightly? Is it just the shoulder level and scapular prominence or are there other factors to this cosmetic perception? The current study has addressed these questions to some measure.

The three domains of shoulder balance may be summarized as radiological, subjective perception (this includes parent's input as well) and objective (clinical photography). The former two domains have been addressed elaborately as per the foregoing discussion. Qui et al. [11] made the first comprehensive study of clinical shoulder asymmetry using clinical photographs. These authors used pre-op photographs and described several tools that may help measure the various shoulder and base of neck elements accurately. However they did not describe the components of torso disfigurement that sometimes occurs after AIS surgery that is the main emphasis of the present study. The TRACE study also utilizes clinical photographs pictured from the back.

From the results of this study it is apparent that shoulder asymmetry has two major components- the base of the neck and the shoulder/scapula/axillary region. Each region has several elements that can individually or collectively affect the aesthetic appeal of that area. For the neck region the tilt of the neck from the vertical axis, the angle of the base of the neck, symmetry and level of trapezius angle and the centrality of the neck upon the shoulder are the chief elements of deformation. In the shoulder region the contributors are the level of the shoulders itself, level and shape of the axillary folds, and the scapular prominence-its size and level. Obviously various permutations and combinations of these are possible and only very large series of cases can represent every possible combination. Fortunately post-operative shoulder imbalance is rare and therefore only large multicenter studies can gather the data required. Radiological correlates to these individual elements has not been attempted in the current study but it would seem interesting to postulate that the neck features would depend more on the $\mathrm{T} 1$ tilt and the shoulder features on the ICL (inter coracoid line). The authors have established in a previous study that the T1 tilt and ICL are often independent of each other [31] and a second prospective study is under way with larger numbers involving pre-operative scoliosis patients addressing their shoulder balance.

The present study has important implications to clinical practice. When comparing pre and post-operative images clinicians can have a more objective tool to document the contrast in shoulder balance. It appears that the base of the neck and the shoulder (including axillary and scapular levels) features determine the aesthetic appeal of the upper trunk region. The upper arm dissymmetry has a lesser role in the body image. Each of these features can independently or collectively cause disfigurement ascribed as post-operative shoulder imbalance. The current understanding of shoulder symmetry may eventually help us prevent shoulder imbalance during AIS surgery.

This study has several limitations. The assessment of aesthetic appeal was performed by two physicians and only consensus was obtained; multiple non-medical judges and statistical tools were not employed. Only posterior views were evaluated for this study. Anterior and lateral images were not studied for socio-cultural reasons. All the photographs were done in the early post op period. It is certainly possible that many of the early shoulder decompensations spontaneously resolved over time though it is believed that this is less likely to occur than trunk imbalance. It is hypothesised that the base of neck features relate to the $\mathrm{T} 1$ tilt and the shoulder features to the ICL (inter coracoid line) but such a radiological correlation was not attempted in this study. The number of cases out of necessity was small and were all operated by one senior surgeon. Obviously larger, multicenter studies looking at different combinations of photographic features and their impact on shoulder cosmesis need to be conducted. 


\section{Conclusions}

Post operative shoulder imbalance consists of neck asymmetry measured by neck axis tilt, neck base obliquity, lack of neck centralization and Trapezius angle asymmetry and, shoulder dissymmetry measured by uneven shoulder levels, axillary fold unevenness, scapular level obliquity and unequal scapular prominence. Each of these two regions can independently affect aesthetic appeal and often exist in combination with other regional asymmetries. Measuring these parameters would objectively document post-operative shoulder imbalance after Scoliosis surgery.

\section{Abbreviations}

AIS: adolescent Idiopathic Scoliosis; SAQ: spinal assessment questionnaire; TAPS: trunk appearance perception scale; TRACE: Trunk Aesthetic Clinical Evaluation; UIV: upper instrumented vertebra; WRVS: Walter Reed Visual Assessment Scale.

\section{Competing interests}

The authors declare that they have no competing interest.

\section{Authors' contributions}

KVM conceived the study, wrote the manuscript and performed most of the surgeries in this series. RKJ and NT collected the cases from one center and HMP and MA from the second center. They collated the clinical photographs and patient outcome data for the study. KVM and NT are part of the "shoulder balance study group" looking at all aspects of shoulder symmetry in scoliosis in the region. KVM and RKJ were the 2 consultants who evaluated the clinical photographs, performed the measurements, tabulated the results and obtained consensus regarding cosmesis. All authors read and approved the submission.

\section{Acknowledgements}

No funds have been received or will be received by any authors of this study.

\section{Author details}

'Orthopaedics, Khoula Hospital, Mina al Fahal, Muscat, Sultanate of Oman. ${ }^{2}$ Department of Neurosurgery, RIPAS Hospital, Bandar Seri Begawan, Brunei Darussalam. ${ }^{3}$ Sparsh Hospital, Bangalore, India. ${ }^{4}$ Amrita Institute of Medical Sciences, Cochin, Kerala, India.

Received: 13 July 2015 Accepted: 8 October 2015

Published online: 17 November 2015

\section{References}

1. Bhandari M, Joensson A, editors. Clinical research for surgeons. Stuttgart: Thieme; 2009. p. 8-9.

2. Kuklo TR, Lenke LG, Graham EJ, Won DS, Sweet FA, Blanke KM, et al. Correlation of radiographic, clinical, and patient assessment of shoulder balance following fusion versus nonfusion of the proximal thoracic curve in adolescent idiopathic scoliosis. Spine. 2002;27(18):2013-20.

3. Ono T, Bastrom TP, Newton PO. Defining 2 components of shoulder imbalance: clavicle tilt and trapezial prominence. Spine. 2012:37(24):E1511-6.

4. Iwahara T, Imai M, Atsuta Y. Quantification of cosmesis for patients affected by adolescent idiopathic scoliosis. Eur Spine J. 1998;7:12-5.

5. Bago J, Carrera L, March B, Villanueva C. Four radiological measures to estimate shoulder balance in scoliosis. J Pediatr Orthop B. 1996:5:31-4.

6. Lee CS, Chung SS, Shin SK, Park YS, Park SJ, Kang KC. Changes of upper thoracic curve and shoulder balance in thoracic adolescent idiopathic scoliosis treated by anterior selective thoracic fusion using VATS. J Spinal Disord Tech. 2011:24:462-8.

7. Yang S, Sean M, Quaidoo J, Eager M, Griffin JW, Reddi V, et al. Right adolescent idiopathic thoracic curve (Lenke $1 \mathrm{~A}$ and B): does cost of instrumentation and implant density improve radiographic and cosmetic parameters? Eur Spine J. 2011;20:1039-47.
8. Cil A, Pekmezci M, Yazici M, Alanay A, Acaroglu RE, Deviren V, et al. The validity of lenke criteria for defining structural proximal thoracic curves in patients with adolescent idiopathic scoliosis. Spine. 2005;30(22):2550-5.

9. Ilharreborde B, Even J, Lefevre Y, Fitoussi F, Presedo A, Souchet P, et al. How to determine the upper level of instrumentation in lenke types 1 and 2 adolescent idiopathic scoliosis; a prospective study of 132 patients. J Pediatr Orthop. 2008;28:733-9.

10. Suk SI, Kim WJ, Lee CS, Lee SM, Kim JH, Chung ER, et al. Indications of proximal thoracic curve fusion in thoracic adolescent idiopathic scoliosis recognition and treatment of double thoracic curve pattern in adolescent idiopathic scoliosis treated with segmental instrumentation. Spine. 2000;25(18):2342-9.

11. Qiu XS, Ma WW, Li WG, Wang B, Yu Y, Zhu ZZ, et al. Discrepancy between radiographic shoulder balance and cosmetic shoulder balance in adolescent idiopathic scoliosis patients with double thoracic curve. Eur Spine J. 2009;18:45-51.

12. Akel I, Pekmezci M, Hayran M, Genc Y, Kocak O, Derman O, et al. Evaluation of shoulder balance in the normal adolescent population and its correlation with radiological parameters. Eur Spine J. 2008;17:348-54.

13. Winter RB. The Idiopathic double thoracic curve pattern: it's recognition and surgical management. Spine. 1989;14:1287-92.

14. Koch KD, Buchanan R, Birch JG, Morton AA, Gatchel RJ, Browne RH. Adolescents undergoing surgery for idiopathic scoliosis: how physical and psychological characteristics relate to patient satisfaction with the cosmetic result. Spine. 2001;26(19):2119-24.

15. Buchanan R, Birch JG, Morton AA, Browne RH. Do you see what I see? Looking at scoliosis surgical outcomes through orthopedists' eyes. Spine. 2003;28(24):2700-4.

16. Smith PL, Donaldson S, Hedden D, Alman B, Howard A, Stephens D, et al. Parents' and patients' perceptions of postoperative appearance in adolescent idiopathic scoliosis. Spine. 2006;31(20):2367-74.

17. Theologis TN, Jefferson RJ, Simpson AH, Turner-Smith AR, Fairbank JC. Quantifying the cosmetic defect of adolescent idiopathic scoliosis. Spine. 1993;18(7):909-12.

18. Drerup B, Hierholzer E. Back shape measurement using video rasterstereography and three-dimensional reconstruction of spinal shape. Clin Biomech. 1994;9:28-36.

19. Drerup B, Hierholzer E. Assessment of scoliotic deformity from back shape asymmetry using an improved mathematical model. Clin Biomech. 1996;11(7):376-83.

20. Raso VJ, Lou E, Hill DL, Mahood JK, Moreau MJ, Durdle NG. Trunk distortion in adolescent idiopathic scoliosis. J Pediatr Orthop. 1998;18:222-6.

21. Berryman F, Pynsent P, Fairbank J, Disney S. A new system for measuring three-dimensional back shape in scoliosis. Eur Spine J. 2008;17:663-72.

22. Zaina F, Negrini S, Atanasio S. TRACE, a routine clinical tool to evaluate aesthetics in scoliosis patients; development from the Aesthetic Index and repeatability. Scoliosis. 2009;4:3. 1-7.

23. Lenke LG, Bridwell KH, O'Brien MF, Baldus C, Blanke K. Recognition and treatment of the proximal thoracic curve in adolescent idiopathic scoliosis treated with Cotrel-Dubousset instrumentation. Spine. 1994;19(14):1589-97.

24. Hong JY, Suh SW, Modi HN, Yang JH, Park SY. Analysis of factors that affect shoulder balance after correction surgery in scoliosis: a global analysis of all the curvature types. Eur Spine J. 2013;22(6):1273-85.

25. Yagi M, Takemitsu M, Machida M. Clavicle chest cage angle difference (CCAD): A novel predictor of postoperative shoulder imbalance in patient with adolescent idiopathic scoliosis. Spine. 2013:38(12):E705-712.

26. Smyrnis PN, Sekouris N, Papadopoulos G. Surgical assessment of the proximal thoracic curve in adolescent idiopathic scoliosis. Eur Spine J. 2009;18(4):522-30.

27. Sanders JO, Polly DW, Cats-Baril W, Jones JA, Lenke LG, O'Brien MF, et al. Analysis of patient and parent assessment of deformity in idiopathic scoliosis using the Walter Reed Visual Assessment Scale. Spine. 2003;28(18):2158-63.

28. Sanders JO, Harrast JJ, Kuklo TR, Polly DW, Bridwell KH, Diab M, et al. The spinal appearance questionnaire: results of reliability, validity, and responsiveness testing in patients with idiopathic scoliosis. Spine. 2007;32(24):2719-22.

29. Carreon LY, Sanders JO, Polly DW, Sucato DJ, Parent S, Roy-Beaudry M, et al. Spinal appearance questionnaire: factor analysis, scoring, reliability, and validity testing. Spine. 2011;36(18):E1240-4. 
30. Bago J, Sanchez-Raya J, Perez-Grueso FJ, Climent JM. The Trunk Appearance Perception Scale (TAPS): a new tool to evaluate subjective impression of trunk deformity in patients with idiopathic scoliosis. Scoliosis. 2010;25:5-6.

31. Menon KV, Tahasildar N, Pillay H, Selvam M, Kumar RJ. Patterns of shoulder imbalance in adolescent idiopathic scoliosis: A retrospective observational study. J Spinal Disord Tech. 2014;27(7):401-8.

Submit your next manuscript to BioMed Central and take full advantage of:

- Convenient online submission

- Thorough peer review

- No space constraints or color figure charges

- Immediate publication on acceptance

- Inclusion in PubMed, CAS, Scopus and Google Scholar

- Research which is freely available for redistribution 